\title{
UNIFORM APPROXIMATION BY POLYNOMIAL, RATIONAL AND ANALYTIC FUNCTIONS
}

\author{
T. G. HONARY ${ }^{凶}$ and S. MORADI
}

(Received 25 June 2007)

\begin{abstract}
Let $K$ and $X$ be compact plane sets such that $K \subseteq X$. Let $P(K)$ be the uniform closure of polynomials on $K$, let $R(K)$ be the uniform closure of rational functions on $K$ with no poles in $K$ and let $A(K)$ be the space of continuous functions on $K$ which are analytic on $\operatorname{int}(K)$. Define $P(X, K), R(X, K)$ and $A(X, K)$ to be the set of functions in $C(X)$ whose restriction to $K$ belongs to $P(K), R(K)$ and $A(K)$, respectively. Let $S_{0}(A)$ denote the set of peak points for the Banach function algebra $A$ on $X$. Let $S$ and $T$ be compact subsets of $X$. We extend the Hartogs-Rosenthal theorem by showing that if the symmetric difference $S \Delta T$ has planar measure zero, then $R(X, S)=R(X, T)$. Then we show that the following properties are equivalent:
\end{abstract}

(i) $\quad R(X, S)=R(X, T)$;

(ii) $\quad S \backslash T \subseteq S_{0}(R(X, S))$ and $T \backslash S \subseteq S_{0}(R(X, T))$;

(iii) $\quad R(K)=C(K)$ for every compact set $K \subseteq S \Delta T$;

(iv) $\quad R(X, S \cap \bar{U})=R(X, T \cap \bar{U})$ for every open set $U$ in $\mathbb{C}$;

(v) for every $p \in X$ there exists an open disk $D_{p}$ with centre $p$ such that

$$
R\left(X, S \cap \bar{D}_{p}\right)=R\left(X, T \cap \bar{D}_{p}\right)
$$

We prove an extension of Vitushkin's theorem by showing that the following properties are equivalent:

(i) $\quad A(X, S)=R(X, T)$;

(ii) $\quad A(X, S \cap \bar{D})=R(X, T \cap \bar{D})$ for every closed disk $\bar{D}$ in $\mathbb{C}$;

(iii) for every $p \in X$ there exists an open disk $D_{p}$ with centre $p$ such that

$$
A\left(X, S \cap \bar{D}_{p}\right)=R\left(X, T \cap \bar{D}_{p}\right)
$$

2000 Mathematics subject classification: 46J10, 46J15.

Keywords and phrases: uniform algebras, polynomial and rational approximation, peak points, planar measure, Vitushkin's theorem.

\section{Introduction}

The algebra of all continuous complex-valued functions on the compact Hausdorff space $X$ is denoted by $C(X)$. The subalgebra $A \subseteq C(X)$ is a Banach function algebra

(c) 2008 Australian Mathematical Society 0004-9727/08 \$A2.00+0.00 
on $X$ if $A$ separates the points of $X$, contains the constants and is complete under an algebra norm. If the norm of a Banach function algebra is the uniform norm then it is a uniform algebra.

Let $A$ be a Banach function algebra on $X$. A point $p \in X$ is a peak point for $A$ if there exists $f \in A$ such that $f(p)=1$ and $|f(x)|<1$ for every $x \in X$ different from $p$. The set of all peak points for $A$ is denoted by $S_{0}(A)$.

Let $K, S, T$ and $X$ be compact subsets of $\mathbb{C}$ such that $K, S, T \subseteq X$, and let $P_{0}(K)$, $R_{0}(K)$ be the algebras of all polynomials and rational functions on $K$ with poles off $K$, respectively. The uniform closures of $P_{0}(K)$ and $R_{0}(K)$ are denoted by $P(K)$ and $R(K)$, respectively, which are uniform algebras on $K$.

The polynomial convex hull of $K$ is

$$
\widehat{K}=\left\{z \in \mathbb{C}:|p(z)| \leq\|p\|_{K} \text { for all polynomials } p\right\} .
$$

The set $K$ is polynomially convex if $\widehat{K}=K$. Let $m$ denote the planar measure and $M(X)$ denote the space of all regular complex Borel measures on $X$. A theorem due to Hartogs and Rosenthal asserts that $R(K)=C(K)$ if $K$ has planar measure zero; see, for example, [2, II.8.4] or [4]. It is also known that $R(K)=C(K)$ if and only if every point of $K$ is a peak point for $R(K)$ [6,5.3.8]. A stronger result is Bishop's peak point criterion for rational approximation, which asserts that if $m(K)=m\left(S_{0}(R(K))\right)$ then $R(K)=C(K)$ [2, II.11.4]. Moreover, $P(K)=R(K)$ if and only if $K$ is polynomially convex. Also a theorem due to Vitushkin gives criteria for $R(K)=C(K)$; see, for example, [2, VIII.5.1] or [7].

In this work we extend the above results to more general algebras in the theory of uniform algebras. For another extension of Hartogs-Rosenthal to Lipschitz algebras, see [5].

If we take $P_{0}(X, K)=\left\{f \in C(X):\left.f\right|_{K} \in P_{0}(K)\right\}$ and $R_{0}(X, K)=\{f \in C(X)$ : $\left.\left.f\right|_{K} \in R_{0}(K)\right\}$ then it is easy to see that $P(X, K)=\left\{f \in C(X):\left.f\right|_{K} \in P(K)\right\}$ and $R(X, K)=\left\{f \in C(X):\left.f\right|_{K} \in R(K)\right\}$ are, in fact, the uniform closures of $P_{0}(X, K)$ and $R_{0}(X, K)$, respectively. We take $A(X, K)=\left\{f \in C(X):\left.f\right|_{K} \in A(K)\right\}$ where $A(K)$ is the algebra of continuous functions on $K$, which are analytic on $\operatorname{int}(K)$. Note that if $K$ is finite then $P_{0}(X, K)=R_{0}(X, K)=C(X)$ and so $P(X, K)=R(X, K)=$ $A(X, K)=C(X)$. Hence, we may assume that $K$ is infinite.

It is easy to show that $P(X, K), R(X, K)$ and $A(X, K)$ are uniform algebras on $X$. Moreover, $P_{0}(X, K)=P_{0}(X), R_{0}(X, K)=R_{0}(X), P(X, K)=P(X), R(X, K)=$ $R(X)$ and $A(X, K)=A(X)$ if $K=X$.

\section{Polynomial and rational approximation in uniform algebras}

Throughout this section we always assume that $K, S, T$ and $X$ are compact plane sets such that $K, S, T \subseteq X$, and $\mu \in M(X)$.

LEMmA 2.1. If $A=\left\{f \in C(X):\left.f\right|_{K}=0\right\}$, then $C_{0}(X \backslash K)=\left.A\right|_{X \backslash K}$. 
ProOF. Clearly, for every $f \in A,\left.f\right|_{X \backslash K} \in C_{0}(X \backslash K)$.

Let $f_{0} \in C_{0}(X \backslash K)$. We extend $f_{0}$ to $X$ by

$$
f(x)= \begin{cases}f_{0}(x), & x \in X \backslash K, \\ 0, & x \in K .\end{cases}
$$

We now show that $f \in A$. Let $x_{0} \in X$. If $f\left(x_{0}\right) \neq 0$ then $x_{0} \in X \backslash K$. Hence, there exists $\delta_{1}>0$ such that $B\left(x_{0} ; \delta_{1}\right) \cap K=\emptyset$. Since $f_{0} \in C_{0}(X \backslash K)$ for every $\varepsilon>0$ there exists $\delta_{2}>0$ such that for every $x \in X \backslash K$ if $\left|x-x_{0}\right|<\delta_{2}$ then $\left|f(x)-f\left(x_{0}\right)\right|<\varepsilon$. If we take $\delta=\min \left\{\delta_{1}, \delta_{2}\right\}$, then for every $x \in X$ if $\left|x-x_{0}\right|<\delta$ then $\left|f(x)-f\left(x_{0}\right)\right|<\varepsilon$. This shows that $f$ is continuous at $x_{0}$.

If $f\left(x_{0}\right)=0$ then for every $\varepsilon>0$ the set $S=\{x \in X \backslash K: f(x) \geq \varepsilon\}$ is compact. Since $x_{0} \in X \backslash S$ there exists $\delta>0$ such that $B\left(x_{0} ; \delta\right) \cap S=\emptyset$. Hence, for every $x \in X \backslash S$, the inequality $\left|f(x)-f\left(x_{0}\right)\right|<\varepsilon$ holds, and this shows that for every $x \in B\left(x_{0} ; \delta\right) \cap X,\left|f(x)-f\left(x_{0}\right)\right|<\varepsilon$ and so $f$ is continuous at $x_{0}$.

LEMMA 2.2. Let $\mu$ be a regular complex Borel measure on $X$. If $U$ is an open set in $\mathbb{C}$ such that for almost all $z \in U$, with respect to planar measure, $\int_{X} d \mu(\zeta) /(\zeta-z)=0$, then $\mu=0$ on $U \cap X$.

Proof. Since $\mu \in M(X)$ it is enough to show that $|\mu|(Y)=0$ for every compact subset $Y$ of $U \cap X$. We consider a decreasing sequence of bounded open neighbourhoods $\left\{U_{n}\right\}_{n=1}^{\infty}$ of $Y$ such that $\bigcap_{n=1}^{\infty} U_{n}=Y$ and $U_{1} \subseteq U$. It is known that for every $n$ we can find a continuously differentiable function $h_{n}$ on the complex plane such that $h_{n}=1$ on $Y, 0 \leq h_{n} \leq 1$ and $E_{n}=\operatorname{supp}\left(h_{n}\right)$ is contained in $U_{n}$. Now let $f \in C^{1}(Y)$, where $C^{1}(Y)$ is the algebra of all continuously differentiable functions on $Y$. We can extend $f$ to a function $g \in C^{1}(\mathbb{C})$ such that it is bounded on the closure of $U_{1}$. Now we define $f_{n}=g h_{n}$. Clearly $f_{n} \in C^{1}(\mathbb{C})$ and it is, in fact, an extension of $f$. By applying Green's theorem as well as Fubini's theorem,

$$
\begin{aligned}
\int_{X} f_{n}(\lambda) d \mu(\lambda) & =\int_{X}\left\{\iint_{E_{n}} \frac{-1}{\pi}(z-\lambda)^{-1}\left(f_{n}\right)_{\bar{z}} d x d y\right\} d \mu(\lambda) \\
& =\iint_{E_{n}} \frac{-\left(f_{n}\right)_{\bar{z}}}{\pi}\left(\int_{X} \frac{d \mu(\lambda)}{z-\lambda}\right) d x d y=0 .
\end{aligned}
$$

Since $\lim _{n \longrightarrow \infty} f_{n}(x)=\chi_{Y}(x) g(x)$, for every $x \in X$, then $\int_{Y} f(\lambda) d \mu(\lambda)=0$ by the dominated convergence theorem. Since the algebra of all such functions $f$ is dense in $C(Y)$, we conclude that $\mu$ is the zero measure on $Y$ and hence $|\mu|(Y)=0$. By the regularity of $\mu$ we conclude that $\mu=0$ on $U \cap X$.

THEOREM 2.3. If $m(S \backslash T)=0$ then $R(X, T) \subseteq R(X, S)$.

Proof. Let $\mu \in(R(X, S))^{\perp}$. We prove that $\mu \in(R(X, T))^{\perp}$. 
We first show that $\operatorname{supp}(\mu) \subseteq S$. For every $f_{0} \in C_{0}(X \backslash S)$ the function

$$
f(x)= \begin{cases}f_{0}(x), & x \in X \backslash S, \\ 0, & x \in S,\end{cases}
$$

belongs to $R(X, S)$ by Lemma 2.1 , hence $\int_{X} f d \mu=\int_{X \backslash S} f_{0} d \mu=0$. Therefore, $\left.\mu\right|_{X \backslash S} \in\left(C_{0}(X \backslash S)\right)^{\perp}$, that is, $\left.\mu\right|_{X \backslash S}=0$. This shows that $\operatorname{supp}(\mu) \subseteq S$.

There exists a bounded open set $U$ such that $U \cap T=\emptyset$ and $X \backslash T \subseteq U$. For every $\alpha \in X \backslash(S \cup T)$, there exists a function $f$ in $R(X, S)$ such that $\left.f\right|_{S}=(z-\alpha)^{-1}$. Hence,

$$
\int_{X}(z-\alpha)^{-1} d \mu=\int_{S}(z-\alpha)^{-1} d \mu=\int_{S} f d \mu=\int_{X} f d \mu=0 .
$$

Since $m(S \backslash T)=0$, for almost all $\alpha \in U, \int_{X}(z-\alpha)^{-1} d \mu(z)=0$. Hence, by Lemma 2.2, $\mu=0$ on $X \cap U=X \backslash T$ and so $\operatorname{supp}(\mu) \subseteq T$. This shows that $\operatorname{supp}(\mu)$ $\subseteq S \cap T$.

Now suppose that $\alpha \in \mathbb{C} \backslash T$. Since $m(S \backslash T)=0, S \backslash T$ has no interior. Hence, there is a sequence $\left\{\alpha_{n}\right\}$ in $\mathbb{C} \backslash S$ such that $\lim _{n \rightarrow \infty} \alpha_{n}=\alpha$.

By hypothesis, $\int_{S \cap T}\left(z-\alpha_{n}\right)^{-1} d \mu=0$ for every $n$. By the dominated convergence theorem,

$$
\int_{S \cap T}(z-\alpha)^{-1} d \mu=\lim _{n \rightarrow \infty} \int_{S \cap T}\left(z-\alpha_{n}\right)^{-1} d \mu=0 .
$$

On the other hand, for every $g \in R_{0}(X, T),\left.g\right|_{T} \in R_{0}(T)$. Since $\left.g\right|_{T}$ is the limit of a sequence of rational functions with poles off $S$, by the same argument as above we conclude that $\int_{S \cap T} g d \mu=0$, and hence

$$
\int_{X} g d \mu=\int_{S \cap T} g d \mu=0 .
$$

Thus for every $g \in R(X, T), \int_{X} g d \mu=0$, that is, $\mu \in(R(X, T))^{\perp}$. Therefore, $R(X, T) \subseteq R(X, S)$.

Corollary 2.4. If $m(S \Delta T)=0$ then $R(X, S)=R(X, T)$.

Corollary 2.5. If $m(K)=0$ then $R(X, K)=C(X)$. In particular, if $m(X)=0$ then $R(X)=C(X)$, which is the Hartogs-Rosenthal theorem.

Proof. Take $S=K$ and $T=\left\{z_{0}\right\}$ for some $z_{0} \in X$, in Corollary 2.4.

Corollary 2.6. If $m(X)=m(K)$ then $R(X, K)=R(X)$.

Proof. Take $S=K$ and $T=X$ in Corollary 2.4.

Theorem 2.7. $R(X, T) \subseteq R(X, S)$ if and only if $\operatorname{supp}(\mu) \subseteq S \cap T$ for every $\mu \in$ $R(X, S)^{\perp}$. 
Proof. Let $R(X, T) \subseteq R(X, S)$ and $\mu \in R(X, S)^{\perp}$. For every $f_{0} \in C_{0}(X \backslash S)$, the function

$$
f(x)= \begin{cases}f_{0}(x), & x \in X \backslash S \\ 0, & x \in S\end{cases}
$$

is continuous on $X$ by Lemma 2.1, and hence $f \in R_{0}(X, S)$. Therefore, $\int_{X \backslash S} f_{0} d \mu=$ $\int_{X} f d \mu=0$, which shows that $\left.\mu\right|_{X \backslash S} \in C_{0}(X \backslash S)^{\perp}$ and so $\left.\mu\right|_{X \backslash S}=0$, that is, $\operatorname{supp}(\mu) \subseteq S$. Since $R(X, S)^{\perp} \subseteq R(X, T)^{\perp}, \mu \in R(X, T)^{\perp}$. Hence, by the same argument as above, $\operatorname{supp}(\mu) \subseteq T$. Therefore, $\operatorname{supp}(\mu) \subseteq S \cap T$.

For the converse, we first show that $\operatorname{int}(S \backslash T)=\emptyset$ if $\operatorname{supp}(\mu) \subseteq S \cap T$ for all $\mu \in R(X, S)^{\perp}$. Suppose on the contrary that there exists a closed disk $D \subseteq \operatorname{int}(S \backslash T)$. Since $R(D) \neq C(D)$ there exists $\lambda \in R(D)^{\perp}$ such that $\lambda \neq 0$. We define the measure $\mu \in M(X)$ by $\mu(E)=\lambda(E \cap D)$, which is not the zero measure. If $f \in R(X, S)$, then

$$
\int_{X} f d \mu=\int_{D} f d \mu=\int_{D} f d \lambda=0
$$

since $\left.f\right|_{D} \in R(D)$. Therefore, $\mu \in R(X, S)^{\perp}$ while $\operatorname{supp}(\mu) \subseteq D \subset S \backslash T$, which is in contradiction with our hypothesis.

Now let $f \in R_{0}(X, T)$ be such that $\left.f\right|_{T}=1 /\left(z-z_{0}\right)$ where $z_{0} \in \mathbb{C} \backslash T$. If $z_{0} \in S \backslash T$ then there exists $\left\{z_{n}\right\} \subset X \backslash(S \cup T)$ such that $\lim _{n \rightarrow \infty} z_{n}=z_{0}$, since $\operatorname{int}(S \backslash T)=\emptyset$. By the dominated convergence theorem,

$$
\lim _{n \longrightarrow \infty} \int_{S \cap T}\left(z-z_{n}\right)^{-1} d \mu(z)=\int_{S \cap T}\left(z-z_{0}\right)^{-1} d \mu(z) .
$$

For every $n \in \mathbb{N}$ there exists $g_{n} \in R_{0}(X, S)$ such that $\left.g_{n}\right|_{S}=\left(z-z_{n}\right)^{-1}$. Since

$$
0=\int_{X} g_{n} d \mu=\int_{S \cap T}\left(z-z_{n}\right)^{-1} d \mu,
$$

it follows that $\int_{S \cap T}\left(z-z_{0}\right)^{-1} d \mu=0$ and so

$$
\int_{X} f d \mu=\int_{S \cap T}\left(z-z_{0}\right)^{-1} d \mu=0 .
$$

If $f$ is an arbitrary element of $R_{0}(X, T)$ then $\left.f\right|_{T \cap S}$ is the limit of a sequence of rational functions with poles off $S \cup T$. Hence, by the above discussion and the dominated convergence theorem, $\int_{X} f d \mu=0$. This shows that $\mu \in R(X, T)^{\perp}$ and so $R(X, T) \subseteq R(X, S)$.

Corollary 2.8. $R(X, S)=R(X, T)$ if and only if $\operatorname{supp}(\mu) \subseteq S \cap T$ for every $\mu \in$ $R(X, S)^{\perp} \cup R(X, T)^{\perp}$.

Theorem 2.9. $R(X, T) \subseteq R(X, S)$ if and only if $S \backslash T \subseteq S_{0}(R(X, S))$. 
Proof. Let $R(X, T) \subseteq R(X, S)$ and $z_{0} \in S \backslash T$. Let $U$ be an arbitrary neighbourhood of $z_{0}$ and let $V$ be a bounded neighbourhood of $z_{0}$ which is contained in $U$ and moreover, $V \cap T=\emptyset$. There exists a neighbourhood $W$ of $z_{0}$ such that its closure is contained in $V$. By Urysohn's lemma there exists a continuous function $f$ on $X$ such that

$$
f(x)= \begin{cases}1, & x \in W, \\ 0, & x \in \mathbb{C} \backslash V .\end{cases}
$$

Since $f$ is zero on $T$ it follows that $f \in R_{0}(X, T)$. Moreover, $|f|<1 / 4$ on $X \backslash U$ and $f\left(z_{0}\right)=\|f\|=1$. Thus $z_{0}$ is a peak point for $R(X, T)$, by $[6,4.7 .22]$. Hence, $S \backslash T \subseteq S_{0}(R(X, S))$.

For the converse, we first note that $\operatorname{int}(S \backslash T)=\emptyset$ by the hypothesis. By Theorem 2.7 it is sufficient to show that $\operatorname{supp}(\mu) \subseteq S \cap T$ for every $\mu \in R(X, S)^{\perp}$. By the same argument as in the proof of Theorem 2.7, it follows that $\operatorname{supp}(\mu) \subseteq S$. We now show that $\left.\mu\right|_{S \backslash T}=0$. For every compact subset $Y$ of $S \backslash T$ there exists a bounded neighbourhood $U$ of $Y$ such that $\bar{U} \cap T=\emptyset$. For every $z_{0} \in U \backslash S$ there exists an $f \in R_{0}(X, S)$ such that $\left.f\right|_{S}=1 /\left(z-z_{0}\right)$. Since $\operatorname{supp}(\mu) \subseteq S$,

$$
\int_{X} \frac{d \mu(z)}{z-z_{0}}=0
$$

If $m(S \backslash T)=0$ then for almost all $z_{0} \in U$,

$$
\int_{X} \frac{d \mu(z)}{z-z_{0}}=0
$$

Hence, by Lemma 2.2, $\mu=0$ on $U \cap X$ and so $\mu=0$ on $Y$. This implies that $\left.\mu\right|_{S \backslash T}=0$.

Now let $m(S \backslash T)>0$. There is a bounded neighbourhood $U$ of $S \backslash T$ such that $U \cap T=\emptyset$. For every

$$
z_{0} \in U \backslash S, \quad \int_{X} \frac{d \mu(z)}{z-z_{0}}=0 .
$$

If for almost all

$$
z_{0} \in U, \quad \int_{X} \frac{d \mu(z)}{z-z_{0}}=0
$$

then, by Lemma 2.2, $\mu=0$ on $U \cap X$ and hence $\left.\mu\right|_{S \backslash T}=0$. Suppose, on the contrary, there exists a compact subset $Y$ of $U$ such that $m(Y)>0$ and for every

$$
z_{0} \in Y, \quad \int_{X} \frac{d \mu(z)}{z-z_{0}} \neq 0 .
$$

Hence, by [6, 5.3. Lemma 1], there exists $z_{0} \in S \backslash T$ such that

$$
\int_{X}\left|z-z_{0}\right|^{-1} d|\mu|(z)<\infty \text { and } \int_{X}\left(z-z_{0}\right)^{-1} d \mu(z) \neq 0 .
$$

We may assume that $\int_{X}\left(z-z_{0}\right)^{-1} d \mu(z)=1$. 
For every

$$
f \in R_{0}(X, S), \quad \frac{f(z)-f\left(z_{0}\right)}{z-z_{0}} \in R_{0}(S) .
$$

By the Tietze extension theorem there exists $F \in R_{0}(X, S)$ such that for every $z \in S$,

$$
F(z)=\frac{f(z)-f\left(z_{0}\right)}{z-z_{0}},
$$

and hence

$$
\int_{X} \frac{f(z)}{z-z_{0}} d \mu(z)=\int_{X} \frac{f\left(z_{0}\right)}{z-z_{0}} d \mu(z)=f\left(z_{0}\right) .
$$

Therefore, for every

$$
f \in R(X, S), \quad \int_{X} \frac{f(z)}{z-z_{0}} d \mu(z)=f\left(z_{0}\right)
$$

by the density of $R_{0}(X, S)$ in $R(X, S)$. Since $z_{0}$ is a peak point for $R(X, S)$, there exists $g \in R(X, S)$ such that $g\left(z_{0}\right)=1$ and $|g|<1$ on $X \backslash\left\{z_{0}\right\}$. For every $n \in N$,

$$
\int_{X} \frac{g^{n}(z)}{z-z_{0}} d \mu(z)=g^{n}\left(z_{0}\right)=1 .
$$

Since for every $z \in X \backslash\left\{z_{0}\right\}, \lim _{n \longrightarrow \infty} g^{n}(z)=0$, by the dominated convergence theorem,

$$
\lim _{n \rightarrow \infty} \int_{X} \frac{g^{n}(z)}{z-z_{0}} d \mu(z)=0,
$$

which is a contradiction. Hence, $\left.\mu\right|_{S \backslash T}=0$, which implies that $\operatorname{supp}(\mu) \subseteq S \cap T$.

Corollary 2.10. $R(X, S)=R(X, T)$ if and only if $S \backslash T \subseteq S_{0}(R(X, S))$ and $T \backslash S \subseteq S_{0}(R(X, T))$.

Corollary 2.11. $R(X, K)=R(X)$ if and only if $X \backslash K \subseteq S_{0}(R(X))$.

PROOF. In Corollary 2.10 we take $S=K$ and $T=X$.

Corollary 2.12. $R(X, K)=C(X)$ if and only if $K \subseteq S_{0}(R(X, K))$. In particular, $R(X)=C(X)$ if and only if $X=S_{0}(R(X))$.

PROOF. In Corollary 2.10 we take $S=K$ and $T=\emptyset$.

Corollary 2.13. If $R(X, S)=R(X, T)$ then $R(X, S)=R(X, T)=R(X, S \cap T)$.

PROOF. This is immediate. 
Theorem 2.14. $P(X, T) \subseteq P(X, S)$ if and only if $\operatorname{supp}(\mu) \subseteq S \cap T$ for every $\mu \in$ $P(X, S)^{\perp}$.

Proof. Let $P(X, T) \subseteq P(X, S)$. By the same method as in the proof of Theorem 2.7, $\operatorname{supp}(\mu) \subseteq S \cap T$ for every $\mu \in P(X, S)^{\perp}$.

For the converse, let $\mu \in P(X, S)^{\perp}$. By hypothesis, $\operatorname{supp}(\mu) \subseteq S \cap T$. For every $f \in P_{0}(X, T),\left.f\right|_{S \cap T}=p$ where $p \in P_{0}(S \cap T)$. If we consider $p$ as an element of $P_{0}(X, S)$ then

$$
\int_{X} f d \mu=\int_{S \cap T} f d \mu=\int_{S \cap T} p d \mu=\int_{X} p d \mu=0 .
$$

This shows that $\mu \in P(X, T)^{\perp}$. Hence, $P(X, T) \subseteq P(X, S)$.

Corollary 2.15. $P(X, S)=P(X, T)$ if and only if $\operatorname{supp}(\mu) \subseteq S \cap T$ for every $\mu \in P(X, S)^{\perp} \cup P(X, T)^{\perp}$.

Corollary 2.16. $P(X, K)=P(X)$ if and only if $\operatorname{supp}(\mu) \subseteq K$ for every $\mu \in P(X)^{\perp}$.

PROOF. In Corollary 2.15 take $S=K$ and $T=X$.

Corollary 2.17. If $P(X, S)=P(X, T)$ then $P(X, S)=P(X, T)=P(X, S \cap T)$.

Proof. We prove that $P(X, S)=P(X, S \cap T)$. Obviously $P(X, S) \subseteq P(X, S \cap T)$. Let $\mu \in P(X, S)^{\perp}$. By Corollary 2.15, $\operatorname{supp}(\mu) \subseteq S \cap T$. For every $f \in P_{0}(X, S \cap T)$, $\left.f\right|_{S \cap T}=p$ where $p \in P_{0}(S \cap T)$. If we consider $p$ as an element of $P_{0}(X, S)$, then $\int_{X} f d \mu=\int_{S \cap T} f d \mu=\int_{S \cap T} p d \mu=\int_{X} p d \mu=0$.

This shows that $\mu \in P(X, S \cap T)^{\perp}$ and hence $P(X, S \cap T) \subseteq P(X, S)$.

Theorem 2.18. $P(X, S)=R(X, T)$ if and only if $S \cap T$ is polynomially convex, $T \backslash S \subseteq S_{0}(R(X, T))$ and $\operatorname{supp}(\mu) \subseteq S \cap T$ for every $\mu \in P(X, S)^{\perp}$.

Proof. Let $P(X, S)=R(X, T)$. So $T \backslash S \subseteq S_{0}(P(X, S))=S_{0}(R(X, T))$. If $\mu \in P(X, S)^{\perp}$ then $\operatorname{supp}(\mu) \subseteq S$ and, since $P(X, S)=R(X, T)$, then $\mu \in R(X, T)^{\perp}$, which implies that $\operatorname{supp}(\mu) \subseteq S \cap T$. By Corollaries 2.15 and 2.8, $P(X, S)$ $=P(X, S \cap T)$ and $R(X, T)=R(X, S \cap T)$ so that $P(X, S \cap T)=R(X, S \cap T)$ and hence $P(S \cap T)=R(S \cap T)$. Therefore $S \cap T$ is polynomially convex.

Conversely, by Corollaries 2.15 and 2.10, $P(X, S)=P(X, S \cap T)$ and $R(X, T)$ $=R(X, S \cap T)$. Since $S \cap T$ is polynomially convex, $P(X, S \cap T)=R(X, S \cap T)$ and hence $P(X, S)=R(X, T)$.

Corollary 2.19. $P(X, K)=R(X)$ if and only if $\widehat{K}=K$ and $X \backslash K \subseteq S_{0}(R(X))$.

PRoOF. In Theorem 2.18 take $S=K$ and $T=X$. 
Corollary 2.20. $P(X)=R(X, K)$ if and only if $\widehat{K}=K$, and for every $\mu \in P(X)^{\perp}, \operatorname{supp}(\mu) \subseteq K$.

PROOF. This is immediate.

Corollary 2.21. If $P(X, K)=R(X)$ and $S$ is a compact subset of $K$ such that $m(S)=m(K)$ then $\widehat{S}=S$.

Proof. By Corollary 2.19, $\widehat{K}=K$. So $P(X, K)=R(X, K)$. By Corollary 2.4, $R(X, K)=R(X, S)$, hence $P(X, K)=R(X, S)$. Therefore, by Theorem 2.18, $\widehat{S}=(\widehat{K \cap S})=K \cap S=S$.

Lemma 2.22. If $P(X, S)=P(X, T)$ then $R(X, S)=R(X, T)$.

Proof. If $P(X, S)=P(X, T)$ then, by the same argument as in the first part of the proof of Theorem 2.9, $S \backslash T \subseteq S_{0}(P(X, S)) \subseteq S_{0}(R(X, S))$ and $T \backslash S \subseteq$ $S_{0}(P(X, T)) \subseteq S_{0}(R(X, T))$. By Corollary 2.10, $R(X, S)=R(X, T)$.

However, the converse of the above lemma is not true. For example, if $X=\{z \in \mathbb{C}:|z|=1\}$ and $S=\{z \in X: \operatorname{Re} z \geq 0\}$ and $T=X$ then $R(X, S)=$ $R(X, T)$, but $P(X, S)=C(X) \neq P(X)=P(X, T)$.

\section{Extension of Vitushkin's theorem}

In 1967 Vitushkin obtained criteria for the equality $R(X)=C(X)$ involving analytic capacity. See [7] or, for example, [2, VIII.5.1]. In this section we extend Vitushkin's theorem.

The following lemma is known; see, for example, [2, p. 64].

LEMMA 3.1. If $\left\{K_{n}\right\}_{n=1}^{\infty}$ is a sequence of compact plane sets such that $R\left(K_{n}\right)$ $=C\left(K_{n}\right)$ for all $n$, and $K=\bigcup_{n=1}^{\infty} K_{n}$ is compact, then $R(K)=C(K)$.

THEOREM 3.2. The following assertions are equivalent:

(i) $R(X, S)=R(X, T)$;

(ii) $R(K)=C(K)$ for every compact subset $K \subseteq S \Delta T$;

(iii) for every compact subset $K \subseteq S \Delta T$, and for every open set $D, \gamma(D \backslash K)$ $=\gamma(D)$, where $\gamma$ is the analytic capacity;

(iv) for every compact subset $K \subseteq S \Delta T$, and for almost all $z \in K$ (with respect to the planar measure),

$$
\limsup _{r \rightarrow 0^{+}} \frac{\gamma(\Delta(z ; r) \backslash K)}{r}>0,
$$

where $\Delta(z ; r)$ is the closed disk with centre $z$ and radius $r$. 
Proof. (i) $\longrightarrow$ (ii) Let $R(X, S)=R(X, T)$ and $K$ be a compact subset of $S \Delta T$. Then $S \backslash T \subseteq S_{0}(R(X, S))$ and $T \backslash S \subseteq S_{0}(R(X, T))$. We take $K_{1}=K \cap S$ and $K_{2}=K \cap T$. Then $K_{1} \subseteq S_{0}(R(X, S)) \subseteq S_{0}\left(R\left(X, K_{1}\right)\right)$ and $K_{2} \subseteq S_{0}(R(X, T))$ $\subseteq S_{0}\left(R\left(X, K_{2}\right)\right)$. Therefore, $K_{1} \subseteq S_{0}\left(R\left(K_{1}\right)\right)$ and $K_{2} \subseteq S_{0}\left(R\left(K_{2}\right)\right)$. Hence, $R\left(K_{1}\right)=C\left(K_{1}\right)$ and $R\left(K_{2}\right)=C\left(K_{2}\right)$ by Corollaries 2.10 and 2.12. By the above lemma, $R(K)=C(K)$.

(ii) $\longrightarrow$ (iii) and (iii) $\longrightarrow$ (iv) are immediate by Vitushkin's theorem [7].

(iv) $\longrightarrow$ (i) We prove that $R(X, S)=R(X, S \cap T)$. So it is sufficient to show that $R(S)=R(S, S \cap T)$. Let $z_{0} \in S \backslash T$ and $U$ be a neighbourhood of $z_{0}$ such that $\bar{U} \cap T=\emptyset$. If we take $K=\bar{U} \cap S$ then

$$
\limsup _{r \rightarrow 0^{+}} \frac{\gamma\left(\Delta\left(z_{0} ; r\right) \backslash K\right)}{r}>0 .
$$

There exists $r_{0}>0$ such that for every $0<r \leq r_{0}, \Delta\left(z_{0} ; r\right) \subseteq U$, so $\Delta\left(z_{0} ; r\right) \backslash K=$ $\Delta\left(z_{0} ; r\right) \backslash S$. Hence,

$$
\limsup _{r \rightarrow 0^{+}} \frac{\gamma\left(\Delta\left(z_{0} ; r\right) \backslash S\right)}{r}>0 .
$$

By Curtis's criterion [2, VIII.4.1], $z_{0}$ is a peak point for $R(S)$. Hence, $S \backslash T \subseteq$ $S_{0}(R(S))$. Therefore, $R(S)=R(S, S \cap T)$ by Corollary 2.11. By the same argument as above $R(X, T)=R(X, S \cap T)$ and hence (i) follows.

In the above theorem we proved that $R(X, S)=R(X, T)$ if and only if for every compact subset $K \subseteq S \Delta T, R(K)=C(K)$. The following example shows that this result and Corollary 2.10 are not true if we replace $R$ by $P$.

Example 3.3. Let $S=C(0 ; 1), T=C(2 ; 1)$ be two circles in the plane and $X=S \cup T$. For every compact subset $K$ of $S \Delta T, K$ is polynomially convex and $m(K)=0$. Hence $P(K)=C(K)$. Moreover, $S \backslash T \subseteq S_{0}(P(X, S))$ and $T \backslash S \subseteq$ $S_{0}(P(X, T))$. If $P(X, S)=P(X, T)$ then $P(X, S)=P(X, S \cap T)=R(X, S \cap T)$ $=R(X, S)$. This implies that $\widehat{S}=S$, which is not true. Therefore, $P(X, S)$ $\neq P(X, T)$.

Corollary 3.4. $R(X, K)=R(X)$ if and only if $R(Y)=C(Y)$ for every compact plane set $Y$ where $Y \subseteq X \backslash K$.

PRoOF. In Theorem 3.2 take $S=K$ and $T=X$.

The next result is, in fact, an extension of a theorem due to Gauthier for compact plane sets; see [3] or [1].

THEOREM 3.5. The following conditions are equivalent:

(i) $R(X, S)=R(X, T)$;

(ii) for every $f \in R(X, S)(R(X, T))$ and for each $\varepsilon>0$ there exists a function $g \in R_{0}(X, T)\left(R_{0}(X, S)\right)$ such that $\|f-g\|_{S \cup T}<\varepsilon$

(iii) for every open set $U$ in $\mathbb{C}, R(X, S \cap \bar{U})=R(X, T \cap \bar{U})$; 
(iv) for every open disk $D$ in $\mathbb{C}, R(X, S \cap \bar{D})=R(X, T \cap \bar{D})$;

(v) for every $p \in X$ there exists an open disk $D_{p}$ with centre $p$ such that

$$
R\left(X, S \cap \bar{D}_{p}\right)=R\left(X, T \cap \bar{D}_{p}\right) .
$$

ProOF. (i) $\longrightarrow$ (ii) is immediate.

(ii) $\longrightarrow$ (i) Let $f \in R(X, S)$ and $\varepsilon>0$. There exists $g \in R_{0}(X, T)$ such that $\|f-g\|_{S \cup T}<\varepsilon / 2$. We can extend $\left.(f-g)\right|_{S \cup T}$ to a continuous function $h$ on $X$ such that $\|h\|_{X}<\varepsilon$. We define $G=f-h$. Then $G \in R_{0}(X, T)$ and $\|f-G\|_{X}$ $=\|h\|_{X}<\varepsilon$. So $f \in R(X, T)$ and hence $R(X, S) \subseteq R(X, T)$. By a similar method, $R(X, T) \subseteq R(X, S)$.

(i) $\longrightarrow$ (iii) $(S \cap \bar{U}) \backslash(T \cap \bar{U}) \subseteq S \backslash T$ and $(T \cap \bar{U}) \backslash(S \cap \bar{U}) \subseteq T \backslash S$. But $S \backslash T$ $\subseteq S_{0}(R(X, S)) \subseteq S_{0}(R(X, S \cap \bar{U}))$ and $T \backslash S \subseteq S_{0}(R(X, T)) \subseteq S_{0}(R(X, T \cap \bar{U}))$. By Corollary 2.10, $R(X, S \cap \bar{U})=R(X, T \cap \bar{U})$.

(iii) $\longrightarrow$ (iv) and (iv) $\longrightarrow$ (v) are immediate.

(v) $\longrightarrow$ (i) We first assume that $K$ is a compact plane set in $S \backslash T$ and then show that $R(K)=C(K)$. For every $p \in K$ there exists $D_{p}$ with a small radius such that $R\left(K_{p}\right)=C\left(K_{p}\right)$ where $K_{p}=K \cap \bar{D}_{p}$. Hence, there are points $p_{1}, p_{2}, \ldots, p_{n}$ in $K$ such that $K \subseteq \bigcup_{i=1}^{n} D_{p_{i}}$ and so $K=\bigcup_{i=1}^{n} K_{p_{i}}$. Since $R\left(K_{p_{i}}\right)=C\left(K_{p_{i}}\right)$ for $i=1,2, \ldots, n$, by Lemma 3.1, $R(K)=C(K)$. Therefore, by Theorem 3.2, $R(X, S)=R(X, S \cap T)$. By the same argument as in the first part of the proof, $R(X, T)=R(X, S \cap T)$. Therefore, $R(X, S)=R(X, T)$.

THEOREM 3.6. $A(X, S)=R(X, T)$ if and only if $\operatorname{int}(S \backslash T)=\emptyset, T \backslash S \subseteq S_{0}(R(X, T))$ and $A(S \cap T)=R(S \cap T)$.

Proof. Let $A(X, S)=R(X, T)$. Clearly $T \backslash S \subseteq S_{0}(R(X, T))$ by Theorem 2.9. If $\operatorname{int}(S \backslash T) \neq \varnothing$ then there exists an open disk $D$ such that $\bar{D} \subseteq S \backslash T$. For every $f \in C(\bar{D})$ there exists an extension $F \in C(X)$ of $f$ such that $F=0$ on $T$. Clearly $F \in R(X, T)=A(X, S)$ and hence $f \in A(\bar{D})$, which is not true.

Since $\operatorname{int}(S \backslash T)=\emptyset$ and $T \backslash S \subseteq S_{0}(R(X, T))$, then $A(X, S)=A(X, S \cap T)$ and $R(X, T)=R(X, S \cap T)$. So $A(X, S \cap T)=R(X, S \cap T)$, which implies that $A(S \cap T)=R(S \cap T)$.

Conversely, since $\operatorname{int}(S \backslash T)=\emptyset$, then $A(X, S)=A(X, S \cap T)$, and moreover, $R(X, T)=R(X, S \cap T)$ by Corollary 2.10. Since $A(S \cap T)=R(S \cap T)$ it follows that $A(X, S \cap T)=R(X, S \cap T)$. Therefore, $A(X, S)=R(X, T)$.

Corollary 3.7. $A(X, S)=R(X, T)$ if and only if for every compact set $K \subseteq$ $T \backslash S, R(K)=C(K)$, for every compact set $K \subseteq S \backslash T, A(K)=C(K)$ and $A(S \cap T)$ $=R(S \cap T)$.

Proof. By Theorem 3.2, $R(X, S \cap T)=R(X, T)$ if and only if $R(K)=C(K)$ for all compact sets $K \subseteq T \backslash S$. On the other hand $R(X, S \cap T)=R(X, T)$ if and only if $T \backslash S \subseteq S_{0}(R(X, T))$, by Corollary 2.10. Since $\operatorname{int}(S \backslash T)=\emptyset$ if and only 
if $A(K)=C(K)$ for all compact sets $K \subseteq S \backslash T$, the result follows from the above theorem.

We are now ready to extend a result due to Boivin and Jiang [1, Theorem 2] for compact plane sets.

THEOREM 3.8. The following assertions are equivalent.

(i) $A(X, S)=R(X, T)$;

(ii) for every closed disk $\bar{D}$ in $\mathbb{C}, A(X, S \cap \bar{D})=R(X, T \cap \bar{D})$;

(iii) for every $p \in X$ there exists a closed disk $\bar{D}_{p}$ in $\mathbb{C}$ with centre $p$ such that $A\left(X, S \cap \bar{D}_{p}\right)=R\left(X, T \cap \bar{D}_{p}\right)$.

Proof. (i) $\longrightarrow$ (ii) By Theorem 3.6, $\operatorname{int}((S \cap \bar{D}) \backslash(T \cap \bar{D}))=\emptyset$, and by Corollary 3.7, $R(K)=C(K)$ for every compact set $K \subseteq(T \cap \bar{D}) \backslash(S \cap \bar{D})$. Since $A(S \cap T)=R(S \cap T)$, it follows from [1, Theorem 2] that

$$
A((S \cap \bar{D}) \cap(T \cap \bar{D}))=R((S \cap \bar{D}) \cap(T \cap \bar{D})) .
$$

So by Corollary 3.7, $A(X, S \cap \bar{D})=R(X, T \cap \bar{D})$.

(ii) $\longrightarrow$ (iii) is immediate.

(iii) $\longrightarrow$ (i) By Theorem 3.6, for each $p \in X, \quad A\left(\left(S \cap \bar{D}_{p}\right) \cap\left(T \cap \bar{D}_{p}\right)\right)$ $=R\left(\left(S \cap \bar{D}_{p}\right) \cap\left(T \cap \bar{D}_{p}\right)\right)$ for a closed disk $\bar{D}_{p}$ in $\mathbb{C}$ with centre $p$. So $A((S \cap T)$ $\left.\cap \bar{D}_{p}\right)=R\left((S \cap T) \cap \bar{D}_{p}\right)$. By [2, II.10.5], $A(S \cap T)=R(S \cap T)$.

Now we prove that $\operatorname{int}(S \backslash T)=\emptyset$. If $p \in \operatorname{int}(S \backslash T)$ then there exists a closed disk $\bar{D}_{p}$ in $\mathbb{C}$ with centre $p$ such that $A\left(X, S \cap \bar{D}_{p}\right)=R\left(X, T \cap \bar{D}_{p}\right)$. Therefore, $\operatorname{int}\left(\left(S \cap \bar{D}_{p}\right) \backslash\left(T \cap \bar{D}_{p}\right)\right)=\emptyset$ by Theorem 3.6. We may take $D_{p}$ with small enough radius such that $\bar{D}_{p} \subseteq S \backslash T$ so that $\bar{D}_{p} \subseteq\left(S \cap \bar{D}_{p}\right) \backslash\left(T \cap \bar{D}_{p}\right)$, which is in contradiction with $\operatorname{int}\left(\left(S \cap \bar{D}_{p}\right) \backslash\left(T \cap \bar{D}_{p}\right)\right)=\emptyset$. Hence, $A(X, S)=A(X, S \cap T)$. By $\quad A\left(X,(S \cap T) \cap \bar{D}_{p}\right)=A\left(X, S \cap \bar{D}_{p}\right)=R\left(X, T \cap \bar{D}_{p}\right), \quad$ it follows that $R\left(X,(S \cap T) \cap \bar{D}_{p}\right) \subseteq R\left(X, T \cap \bar{D}_{p}\right)$, so $R\left(X,(S \cap T) \cap \bar{D}_{p}\right)=R\left(X, T \cap \bar{D}_{p}\right)$. Hence, $R(X, S \cap T)=R(X, T)$ by Theorem 3.5. On the other hand, $A(X, S \cap T)$ $=R(X, S \cap T)$ since $A(S \cap T)=R(S \cap T)$. Therefore, $A(X, S)=R(X, T)$.

\section{References}

[1] A. Boivin and B. Jiang, 'Uniform approximation by meromorphic functions on Riemann surfaces', J. Anal. Math. 93 (2004), 199-214.

[2] T. W. Gamelin, Uniform Algebras (Chelsea Publishing Company, New York, 1984).

[3] P. M. Gauthier, 'Meromorphic uniform approximation on closed subsets of open Riemann surfaces', in: Approximation Theory and Function Analysis, Proc. Conf. Campinas, 1977 (ed. J. B. Prolla) (North-Holland, Amsterdam, 1979), pp. 139-158.

[4] F. Hartogs and A. Rosenthal, 'Über Folgen analytischer Funktionen', Math. Ann. 104 (1931), 606610 .

[5] T. G. Honary and H. Mahyar, 'Approximation in Lipschitz algebras', Quaest. Math. 23(1) (2000), 13-19.

[6] G. M. Leibowitz, Lectures on Complex Function Algebras (Scott, Foresman, Glenview, IL, 1970).

[7] A. G. Vitushkin, 'Analytic capacity of sets and problems in approximation theory', Uspekhi Mat. Nauk. 22 (1967), 141-199 (Engl. Transl. Russian Math. Surveys 22 (1967), 139-200). 
T. G. HONARY, Faculty of Mathematical Sciences and Computer Engineering, Tarbiat Moallem University, 599 Taleghani Avenue, 15618, Tehran e-mail: honary@saba.tmu.ac.ir

S. MORADI, Faculty of Mathematical Sciences and Computer Engineering, Tarbiat Moallem University, 599 Taleghani Avenue, 15618, Tehran e-mail: S_moradi@tmu.ac.ir 